\title{
The Liver Unit at King's College
}

Hepatology has emerged as an increasingly important, dynamic, and exciting discipline during the latter half of the 20th century. Major breakthroughs which fostered the growth of interest in the study of the liver include the development of biochemical tests which allow evaluation of selected functions, the introduction of the percutaneous liver biopsy, the explosive increase in knowledge regarding viruses which affect the liver, and most recently the emergence of liver transplantation as an important therapeutic option for many hitherto untreatable acute and chronic liver diseases.

A few major liver units throughout the world have led the way in providing direction and in the training of leaders for hepatology. The liver unit at King's College Hospital surely fits into this select group and has played a major role in the emergence of modern hepatology. For 25 years the basic science and clinical contributions from the Liver Unit at King's College Hospital, under the capable leadership of $\mathrm{Dr}$ Roger Williams, has had a major impact in the development of our understanding of the liver and its disorders.

The clinicians and basic scientists at King's College Hospital have made contributions across a broad range. Several longterm interests of the group bear special mention including the role of the liver unit in fostering understanding of the pathophysiologic consequences of and approaches to treatment in patients with fulminant hepatocellular failure, the type and importance of immunologic reactions affecting the liver, and the pioneering work of the liver unit in association with Sir Roy Calne at Cambridge in the development of liver transplantation.

Interest in fulminant hepatocellular failure at King's College was fostered by the large numbers of patients who attempted, and occasionally achieved, suicide through taking an overdose of acetaminophen (paracetamol). Major features of the ensuing somewhat predictable syndrome which have been studied include the importance of raised intracranial pressure as a cause of death, the nature of the often profound coagulopathy that develops, characteristics of the hepatic encephalopathy, the determinative role of sepsis, and more recently introduction of liver transplantation as life saving therapy. In addition to recognition and definition of the pathophysiologic consequences of fulminant hepatic failure, many innovative therapeutic approaches have been evaluated in the Unit. Several have helped; others have not stood the test of time but have fostered additional research. Various approaches to reduce intracranial pressure in fulminant hepatic failure include controlled hyperventilation, use of mannitol, use of thiopental, and evaluation of the role of corticosteroids have all been extensively studied. For many years studies from the Unit evaluated the use of charcoal perfusion filtering systems with the goal of removal of toxins across specialised membranes in a patient with a failing liver and kidney. Unfortunately, early apparent success in the treatment of patients with fulminant hepatic failure treated with charcoal perfusion was not confirmed when additional patients were treated. These studies of haemoperfusion have, however, provided important background information for the development of much needed artificial liver support systems.

A second major direction for the group has been in the area of liver transplantation. From the early collaborative efforts of the Liver Unit at King's College with Sir Roy Calne and his colleagues in Cambridge, a productive series of clinical observations and investigations ensued. The group was among the first to appreciate the importance of cyclosporin as an effective immunosuppressive agent. Attention has been directed towards refining prognostic indicators which suggest the most appropriate time for proceeding to liver transplantation in a variety of liver disorders ranging from fulminant hepatic failure to primary biliary cirrhosis. In many areas the King's College-Cambridge Programme has provided leadership in the development of liver transplantation.

A third important area of investigation pursued by the Liver Unit at King's College has focused upon immunologic reactions which affect the liver. The immunologic components of liver injuries studied range from drug induced changes, viral related injury, alcohol induced changes, and evaluation of the role of altered immune responses in patients with idiopathic autoimmune chronic hepatitis and primary biliary cirrhosis. In relation to drugs, several notable areas have been studied including the discovery that patients with halothane induced liver injury have antibodies which are directed against altered cell components. Investigation of these antibodies may yield additional information as to the pathogenesis of this and related drug induced liver injuries. Many studies have been reported from the group regarding the role of liver specific proteins in the pathogenesis of liver disease. In patients with idiopathic autoimmune chronic active hepatitis, the continuing presence of anti-liver specific proteins antibodies was found to predict clinical relapse even if there had been an apparent longterm drug induced remission.

In these brief comments, I have chosen to mention only a few areas of hepatology in which the Liver Unit at King's College Hospital has made major contributions. There are many others we might discuss. These include such diverse subjects as the pathophysiology and treatment of haemochromatosis; the spectrum of liver injury in Budd-Chiari syndrome; the treatment of chronic viral hepatitis with antiviral agents; as well as the study of many types of drug induced liver injuries and expansion of the spectrum of alcohol induced liver disease. An overall assessment of these contributions leaves no doubt that the emergence of modern hepatology has been facilitated by the formation and continued contributions of the Liver Unit at King's College Hospital.

WILLIS C MADDREY

University Medical Centre,

Southwestern Medical Center,

5323 Harry Hines Boulevard,

Dallas, Texas, USA 